\title{
Research on advanced programming based on DLL and API
}

\author{
Yurong Guan ${ }^{1, a}$, Fen Zhou ${ }^{2, b}$ \\ ${ }^{1}$ Huanggang Normal University, Huangzhou, Hubei, China \\ ${ }^{2}$ Huanggang Normal University, Huangzhou, Hubei, China \\ ajsjgyr@hgnu.edu.cn, bjsjzhoufen@hgnu.edu.cn
}

Keywords: API, DLL, VB

Abstract. In the visual development environment, skilled call API and DLL function, combined with the Windows system kernel, in order to make the user experience Windows API function programming ability strong. In this paper, we first introduce the DLL library and API function about the basic knowledge, followed by operating in the network link, image processing, media control, through specific examples of API function to achieve high-level programming skills, and finally illustrates the API and DLL calling conventions. The key is to allow users to advanced programming capability of using Windows API and the DLL function to the daily life and production, not only improve the level of programming, but also provides a simple, efficient and practical service for the people.

\section{Introduction}

As is known to all, the operating system is computer system "housekeeper", in fact, the windows operating system in addition to the coordinated application execution, memory allocation, management of system resources, also is a great "service center", each a "service" actually is equivalent to a function. If these functions are called can realize such as open windows, graphics processing, use peripherals, diagnosis and other powerful processing function, and these functions are called service object is some applications, so called for the application programming interface.

\section{DLL library and API function}

API interface is a group of mainly written in C language function, according to the usual classification standards, the windows API[1] function can be divided into seven categories: window management, common window control, shell characteristics, graphics device interface, system services, international characteristics, network services. It has more than 600 pre compiled functions and procedures, in the installation of Windows system, these functions or processes have been installed, and put in Libraries Dynamic-Link. DLL[2] is a process library, in the run fashion, DLL library update can be carried out independently, but also can be multiple applications to share the same DLL library. The core of the windows about API and DLL has kernel32.dll, gdi32.dll, user32.dll, advapi32.dll, shell32.dll, etc.. DLL is one of the important technical means to realize the sharing of resources, save memory space, solve platform differences and improve the use efficiency of Windows application program. Common dynamic library contains external functions and resources, but also has some dynamic library only contains resources, such as Windows font resource files, this kind of dynamic library called resources dynamic link library. DLL function and process are the external process, must first statement, the following is declare DLL process of syntax, the declare statement "lib" used to find the DLL file that contains the process, such as Win32api.txt, the file is located in the VB under the main directory of the WinAPI subdirectories in, It contains the many windows API declaration of a procedure.

[Public][/Private] declare Function [/Sub] Process name lib "libname" [Alias "name"] [([[ByVal] variable name [as type],...])] As type 
Of course, after the declaration of an external process, you can link and use it at run time, just as in the call to the internal Sub or Function. Note that the Function process has a return value, if you do not need to return the value of the Function process, you can also call the same as the Sub process to write a separate statement.

\section{Application of DLL and API}

In many visualization platform, visual basic language is simple and practical, and windows kernel system perfect binding and unique display advanced features of the DLL and API, with a powerful programming ability, it has three versions [3], can meet the needs of different development. Learning edition enable programmers to easily develop windows and windows NT applications, including all internal controls and the grid, tab and data bound controls, professional edition for professional programmers provides a set of complete function development tools, including ActiveX controls, visual database tools, dynamic html page designer etc.., enterprise edition allows programmers to develop powerful distributed applications, including back office tools, SQL server, microsoft transaction server, internet information server.

Implementation of Internet link

In the realization of the core code, first need to declare the API "ShellExecute" function[4], the method for click the add menu of the add in manager command, will open the add in Manager dialog, this dialog box can be an external program option double-click "VB 6 API Viewer" loading, then click add menu of the "API browser" command, you can open the API browser window, then the window click file menu "loading text file" command, this will pop a select a text API file dialog box, select "Win32api.txt"[5] the file from this window again, available option in the "ShellExecute" function and then double-click Click the copy button, finally opened the established Module1 well, i.e. "ShellExecute" function can be pasted into the Module 1 module code window, and its code is as follows:

Public Declare Function ShellExecute Lib "shell32.dll" Alias "ShellExecuteA" (ByVal hwnd As Long, ByVal lpOperation As String, ByVal lpFile As String, ByVal lpParameters As String, ByVal lpDirectory As String, ByVal nShowCmd As Long) As Long

When after the success of the API function declarations[6], you can write a call "shellexecute" function sub process in the code window, for example, addresslink $=$ shellexecute $(0 \&$, vbNullString, address, vbNullString, vbNullString, vbNormalFocus), while the program is running, the API functions and DLL library function will play role the, user if the network connection is successful, the browser will be able to open the links page.

The application, the user uses object oriented program design method is developed, combined with the network environment of the Internet link, experience the API this a series of the underlying function provides to the user for access to the operating system kernel programming way.

Realization of image filter processing

In daily life, in order to get the picture right color and often using filter processing, simple said is through the different color to observe the picture color changes to achieve image processing of special effects, often used in medical tests, digital photography, sunglasses and so on many aspects. In this design, the main use of the API function is "GetPixel" and "SetPixel", the two API functions related to the setting can be achieved on the image of a monochrome or mixed color filter. The "GetPixel" function is used to obtain the RGB value of a pixel in the specified device scenario, and the "SetPixel" function is used to set the RGB value of a pixel in the specified device context.

Public Declare Function GetPixel Lib "gdi32" Alias "GetPixel" (ByVal hdc As Long, ByVal x As Long, ByVal y As Long) As Long

Public Declare Function SetPixel Lib "gdi32" Alias "SetPixel" (ByVal hdc As Long, ByVal x As Long, ByVal y As Long, ByVal crColor As Long) As Long 
In the implementing code. First of all, the a variable assignment statements Rgbp $=$ GetPixel (PicHdc, I, J), complete the GetPixel function calls, picture of a pixel RGB value is obtained; then through the three primary colors of red, green and blue color of a pixel point were a variety of other color filter processing, a new pixel RGB value is obtained; finally through the procedure call statement "setPixel PicHdc, I, J, Rgbp", achieve setPixel function calls, with the RGB value set of a pixel color can be carried out the corresponding color image filter.

In the implementation, respectively, the red, green and blue monochromatic complete the picture filter, also can complete a variety of different colors mixed filter, and the operation is very simple and convenient, as a professional image processing software as to solve many practical problems, also on the image filter processing process understand more thoroughly, but also improve the ability of programming application.

Implementation of CD play

Use API function to write a CD player to replace the use of multimedia controls, not only the program compiled EXE file size is small, and the efficiency is relatively high. The main use of this API function is "mciSendString". Open the code window, use the "API Viewer" tool can add the "mciSendString" function declaration.

Private Declare Function mciSendString Lib "winmm.dll" Alias "mciSendStringA" (ByVal lpstrCommand As String, ByVal lpstrReturnString As String, ByVal_uReturnLength As Long, ByVal hwndCallback As Long) As Long

The "MciSendString" function statement, you can control program function call play the CD, such as the playback control code for: play1 = mciSendString ("play cdaudio", returnstring, 127 and 0 ), therefore, the user can be one by one in the stop play, pause, open the $\mathrm{CD}$, playing a $\mathrm{CD}$, total number of tracks, current track length control function call, the realization of CD playback.

If you put a $\mathrm{CD}$ record in the $\mathrm{CD}$ drive, and then run, click the play button, the music will be wonderful to play, but you can also more design style shows the track of the relevant information. Windows powerful MCI for the multimedia program design has opened up a vast world, you can make a very good multimedia program.

\section{API and DLL call specifications}

API and DLL are powerful, closely integrated with the Windows system, but there are some matters needing attention in use. Check the variable declaration requirements, before writing code, it is best not to use the variable type coercion, in order to avoid conflict, causing the program can not run normally. Check the parameter type, in the Win32 environment, whether it is 8-bit, 16 bit or 32-bit numeric variables are "wrong DLL call norms" message to 32-bit transfer, if used at the same time, transfer to an API function, will, it is very difficult to find the error. Correctly understand the data type and data structure of API function, and analyze the application environment of DLL. To reduce and avoid using as any statement base method, although this method can make the API function to receive various types of parameters, but also makes the system difficult to examine other data errors, if there is a misuse of the ByVal key word such a small mistake may cause the system to crash. To ensure that the integrity of the function declaration, in the parameters of the transfer, the analysis is the value of the transmission or by address pass, so as to avoid the API Windows function does not support the data type and the error occurred. Check the API function, especially the case sensitive, easy to make these small details in a DLL function to find the function statement or find is not a function of the need. Pre initialization string, if the API function requirements, a pointer to the buffer is, if there is no pre initialization string length, API function there will be possible at the end of the buffer rewrite, leading to the program came to an abrupt halt or intermittent errors. Check the parameters and return type and the return value, the general use of single step operation mode, source and type of track inspection parameters, excluding parameter error transmission, to ensure correct program execution. 


\section{Conclusions}

API is a set of function definition, parameter definition and message format supported by the operating system, in a variety of visual programming platform, if programmers want to write advanced applications to higher efficiency, it is more important than to understand the performance of API and DLL, although the use of built-in programming environment in many libraries and functions are in fact, those easy, classes and functions are built on Windows API, to accelerate the development of Windows applications, but it is only equivalent to touch the Windows system function of fur, programmers only really understand the connotation of API and DLL, to master the powerful function of API function, the library and visual development the environment successfully extended to, in order to develop efficient procedures for better performance.

\section{Acknowledgements}

I thank reviewers for constructive reviews and suggestions that improved the quality of this paper. This essay is the fruit of our several months' consisting work. This work was supported by the scientific research project of Huanggang Normal University.

\section{References}

[1] Skonnard, Aaron, Essential Winlnet: Developing Applications Using the Windows Internet API with Ras, ISAPI, ASP, and Com, Addison-Wesley Professional , December 2011.

[2] Weijiang Jiang, Visual Basic API programming hundred cases pass, Science Press, October 2001.

[3] Information on http://www.chinavb.net.

[4] Dingxue Wu, Visual Basic Programming [M], Beijing: Science Press, February 2008.

[5] Information on http://baike.baidu.com.

[6] Aqi Zheng, Zuofa Peng.Visual Basic.NET programming tutorial [M]. Guangzhou: South China University of Technology press, May 2006. 